\title{
Generation Rescheduling Based on Energy Margin Sensitivity for Transient Stability Enhancement
}

\author{
Kyu-Ho Kim*, Sang-Bong Rhee ${ }^{* *}$, Kab-Ju Hwang***, Kyung-Bin Song ${ }^{\dagger}$ and Kwang Y. Lee ${ }^{\S}$

\begin{abstract}
This paper presents a generation rescheduling method for the enhancement of transient stability in power systems. The priority and the candidate generators for rescheduling are calculated by using the energy margin sensitivity. The generation rescheduling formulates the Lagrangian function with the fuel cost and emission such as $N O_{x}$ and $S O_{x}$ from power plants. The generation rescheduling searches for the solution that minimizes the Lagrangian function by using the Newton's approach. While the Pareto optimum in the fuel cost and emission minimization has a drawback of finding a number of non-dominated solutions, the proposed approach can explore the non-inferior solutions of the multiobjective optimization problem more efficiently. The method proposed is applied
\end{abstract} \\ to a 4-machine 6-bus system to demonstrate its effectiveness.
}

Keywords: Generation rescheduling, Transient stability enhancement, Emission control, Energy margin sensitivity, Newton's approach.

\section{Introduction}

Dynamic security analysis has dealt with the evaluation of the ability of a power system to withstand contingencies, i.e., to remain stable and settle to an acceptable steady-state condition.

Many approaches have been applied for solving the optimal power flow (OPF) problem, including methods of direct optimization of the non-linear OPF problem, such as the Newton's approach which uses a sparsityoriented simultaneous solution method for a quadratic approximation of the Lagrangian function [1-3]. Especially, the preventive security dispatch OPF was used for system stability enhancement [4-7]. Several generation rescheduling strategies for the improvement of transient stability based on energy margin have been proposed where the stability requirement is considered as a constraint for pseudo optimality over a year [8, 9]. Especially, stability limits resulting from plant generation limits, load changes, or network configuration changes are derived using analytical sensitivities of the energy margin [10]. The energy margin sensitivities to system parameters are used to determine new schedules of stability limits by using optimization methods. Sensitivities to certain parameters such as generation limits are built into the system models before the boundary of stability region based controlling unstable equilibrium point (BCU) method is applied [8, $11,12]$.

$\dagger$ Corresponding Author: Dept. of Electrical Engineering, Soongsil

University, Korea. (kbsong@ssu.ac.kr)

* Dept. of Electrical Engineering, Hankyong University, Korea.

** Dept. of Electrical Engineering, Yeungnam University, Korea.

*** Dept. of Electrical Engineering, University of Ulsan, Korea.

$\S \quad$ Dept. of Electrical and Computer Engineering, Baylor University, USA

Received: December 13, 2014; Accepted: September 7, 2015
The generation rescheduling to enhance dynamic security for multi-area power systems consists of dynamic security assessment (DSA) using the transient energy function method, sensitivity analysis, control coordination among the areas, and the generation shift distribution factor (GSDF) [13]. However, in 1995, generation rescheduling was proposed without considering the sensitivities of the energy margin to system parameters, where at the time of fault clearing, the rate of change of speed is brought closer for all generators [9]. Recently, a new generation rescheduling approach is proposed that can optimally reallocate power generations for multiple unstable contingencies for preventive control of power systems [14], where the transient stability constraints used in the optimal rescheduling model are described by a heuristic stability performance index.

In addition, with the increasing concern on environmental pollution, the generation rescheduling considering economic dispatch alone can no longer be the only objective in power system operation. Many schemes have been reported to solve multi-objective environmental/economic power dispatch problems which have drawn considerable attention [15-17].

This paper presents a generation rescheduling method for the enhancement of transient stability in power systems. In order to determine the generation rescheduling priority and candidate generators, the energy margin sensitivity indices are calculated. The selected number of candidate generators instead of all generators can be used as a variable for generation rescheduling and reduce the search space. The generation rescheduling formulates the Lagrangian function with the fuel cost and emission such as $N O_{x}$ and $S O_{x}$ of power plants. The optimal solution that minimizes the Lagrangian function is searched by the 
Newton's approach. While the Pareto optimum in the fuel cost and emission minimization has a number of nondominated solutions, the proposed approach can explore non-inferior solutions of the multiobjective optimization problem more efficiently.

\section{Formulation of Generation Rescheduling with the Newton's Approach}

The generation rescheduling problem consists of minimizing two objective functions, fuel cost and emission pollutants, while satisfying system constraints such as voltage magnitude constraints and power flow limits, etc.

\subsection{Objective functions}

\subsubsection{Fuel cost:}

This objective function is the total fuel cost of the system. The fuel cost curves of the thermal generators are modeled as typical quadratic functions and represented as

$$
F\left(P_{G}\right)=\sum_{i=1}^{N G}\left(a_{i}+b_{i} P_{G i}+c_{i} P_{G i}^{2}\right)
$$

where $N G$ is the number of generators, $a_{i}, b_{i}, c_{i}$, and $P_{G i}$ are, respectively, the cost coefficients and the real power output of the $i^{t h}$ generator. $P_{G}$ is the vector of $N G$ real power outputs of generators, $P_{G}=\left[P_{G 1}, P_{G 2}, \cdots, P_{G N G}\right]$.

\subsubsection{Emission:}

The total emission in $($ ton $/ h)$, of atmospheric pollutants such as sulpher oxides, $S O_{x}$ and nitrogen oxides, $N O_{x}$ caused by from fossil-fueled thermal units can be mathematically modeled as [15-17]

$$
E\left(P_{G}\right)=\sum_{i=1}^{N G} 10^{-2}\left(\alpha_{i}+\beta_{i} P_{G i}+\gamma_{i} P_{G i}^{2}\right)+\zeta_{i} \exp \left(\mu_{i} P_{G i}\right)
$$

where $\alpha_{i}, \beta_{i}, \gamma_{i}, \zeta_{i}$, and $\mu_{i}$ are the emission characteristic coefficients of the $i^{\text {th }}$ generator.

\subsection{Constraints}

\subsubsection{Equality constraints:}

The load, active and reactive power balances of system must be satisfied:

$$
\begin{aligned}
& \sum_{i=1}^{N G} P_{G i}-P_{D}-P_{\text {Loss }}=0 \\
& \Delta P_{j}=0, \quad \Delta Q_{j}=0, \quad j=1, \ldots, N B
\end{aligned}
$$

where $P_{D}$ and $P_{\text {Loss }}$ are the total demand and the real power loss in transmission lines, respectively. $N B$ is the number of system buses. $\Delta P_{j}$ and $\Delta Q_{j}$ are the active and reactive power balances at bus $j$, respectively.

\subsubsection{Inequality constraints:}

The real power output of each generator and the bus voltage magnitude are constrained by each limit, i.e.,

$$
\begin{array}{ll}
P_{G i}^{\min } \leq P_{G i} \leq P_{G i}^{\max }, & i=1, \ldots, N G \\
V_{j}^{\min } \leq V_{j} \leq V_{j}^{\max }, & j=1, \ldots, N B
\end{array}
$$

where $P_{G i}^{\min }$ and $P_{G i}^{\max }$ are the minimum and maximum power output of the $i^{\text {th }}$ power plants, respectively. $V_{j}$ is the voltage magnitude of bus $j . V_{j}^{\min }$ and $V_{j}^{\max }$ are the lower and upper limits of bus voltage magnitude, respectively. The transmission line loading is restricted by its upper limit as

$$
\mid \text { pflow }_{l} \mid \leq \text { pflow }_{l}^{\max }, \quad l=1, \ldots, N L
$$

where $N L$ is the number of transmission lines. pflow and pflow $_{l}^{\max }$ are the active power flow and the thermal limit of line $l$.

\subsection{Newton's approach}

The objective functions and constraints in (1) - (5) can be transformed into a general nonlinear optimization problem, which consists of finding an optimum of a nonlinear objective function subject to a set of equality and inequality constraints. Specifically, the problem can be reformulated as a Lagrangian minimization problem for a given active constraint set described as $(6)[2,3]$.

$$
\begin{array}{ll}
\text { Minimize } & w \cdot F(y)+(1-w) \cdot \Phi \cdot E(y) \\
\text { Subject to } & \left(g_{i}(y)-c_{i}=0\right) \quad i=1,2, \ldots \ldots, m
\end{array}
$$

where $F(y)$ and $E(y)$ are nonlinear objective functions made of objective functions (1) and (2). $y$ is a column vector of $n$ variables, $y=\left[P_{G}, V, \theta\right]^{T} . V$ and $\theta$ are the vector of $N B$ variables for the bus voltage magnitude and angle. $w$ and $\Phi$ are a weight factor and a scaling factor, respectively. $g_{i}(y)-c_{i}=0$ is a nonlinear equation in $y$ with $c_{i}$ as constant of (3) - (5).

The above constrained minimization problem can be converted into an equivalent unconstrained minimization problem with the Lagrangian function

$$
L(y, \lambda)=w \cdot F(y)+(1-w) \cdot \Phi \cdot E(y)-\lambda^{T}\left[\begin{array}{c}
g_{1}(y)-c_{1} \\
g_{2}(y)-c_{2} \\
\vdots \\
g_{m}(y)-c_{m}
\end{array}\right]
$$


where $L(y, \lambda)$ is the Lagrangian function, and $\lambda^{T}$ is the transposed vector of $m$ Lagrange multipliers.

The necessary conditions for a constrained minimum of $F(y)$ and $E(y)$ can be written as:

$$
\begin{aligned}
\frac{\partial L}{\partial y}(y, \lambda)= & w \cdot \frac{\partial F}{\partial y}(y)+(1-w) \cdot \Phi \cdot \frac{\partial E}{\partial y}(y) \\
& -\lambda^{T}\left[\frac{\partial g_{1}}{\partial y}(y) \quad \frac{\partial g_{2}}{\partial y}(y) \quad \cdots \quad \frac{\partial g_{m}}{\partial y}(y)\right]^{T} \\
= & h_{1}(y, \lambda)=0 \\
\frac{\partial L}{\partial \lambda}(y, \lambda)= & -\left[\begin{array}{c}
g_{1}(y)-c_{1} \\
g_{2}(y)-c_{2} \\
\vdots \\
g_{m}(y)-c_{m}
\end{array}\right]=h_{2}(y)=0 \quad i=1,2, \ldots \ldots, m
\end{aligned}
$$

If the Taylor series expansion is used in the previous set of equations in (8) and the higher order terms are neglected, then

$$
\begin{aligned}
& h_{1}(y, \lambda)=h_{1}\left(y_{0}, \lambda_{0}\right)+\left[h_{1}^{\prime}\left(y_{0}, \lambda_{0}\right)\right]^{T}\left[\begin{array}{l}
\Delta y \\
\Delta \lambda
\end{array}\right]=0 \\
& h_{2}(y)=h_{2}\left(y_{0}\right)+h_{2}^{\prime}\left(y_{0}\right) \Delta y=0
\end{aligned}
$$

by assuming $y=y_{0}+\Delta y, \lambda=\lambda_{0}+\Delta \lambda$. Rearranging (9) in a matrix form, we obtain

$$
\left[\begin{array}{cc}
\frac{\partial h_{1}}{\partial y} & \frac{\partial h_{1}}{\partial \lambda} \\
\frac{\partial h_{2}}{\partial \lambda} & 0
\end{array}\right]\left[\begin{array}{c}
\Delta y \\
\Delta \lambda
\end{array}\right]=\left[\begin{array}{c}
-h_{1} \\
-h_{2}
\end{array}\right]
$$

or, from (8),

$$
\left[\begin{array}{cc}
\frac{\partial^{2} L}{\partial y^{2}} & \frac{\partial^{2} L}{\partial y \partial \lambda} \\
\frac{\partial^{2} L}{\partial \lambda \partial y} & 0
\end{array}\right]\left[\begin{array}{l}
\Delta y \\
\Delta \lambda
\end{array}\right]=\left[\begin{array}{c}
-\frac{\partial L}{\partial y} \\
-\frac{\partial L}{\partial \lambda}
\end{array}\right]
$$

Eq. (11) gives the iterative correction vector $\left[\begin{array}{ll}\Delta y & \Delta \lambda\end{array}\right]^{T}$ in applying the Newton's approach for minimizing the Lagrangian function. The set of all first-order partial derivatives of $L(y, \lambda)$ form the gradient vector

$$
\nabla L(y, \lambda)=\left[\begin{array}{l}
\nabla L(y) \\
\nabla L(\lambda)
\end{array}\right]=\left[\begin{array}{l}
\frac{\partial L}{\partial y} \\
\frac{\partial L}{\partial \lambda}
\end{array}\right]
$$

$L(y, \lambda)$ is a vector of $(\mathrm{n}+\mathrm{m})$ functions. The subvector $\nabla L(y)$ is a function of $y$ and $\lambda$, but the subvector $\nabla L(y)$ is a function of $y$ only. The set of all secondorder partial derivatives of $L(y, \lambda)$ forms an $(n+m) \times(n+m) \quad$ symmetric matrix $\nabla^{2} L(y, \lambda)$. Since some of the second-order partial derivatives of $L(y, \lambda)$ can be reduced to the first-order partial derivatives of the constraint equations, $\nabla^{2} L(y, \lambda)$ is rarely being called as the Hessian matrix of $L(y, \lambda)$. This matrix, denoted as $W$, will be used in this paper $[2,3]$. The $W$ matrix can be partitioned into four principal submatrices and is represented by

$$
W(y, \lambda)=\left[\begin{array}{cc}
H(y, \lambda) & -J^{T}(y) \\
-J(y) & 0
\end{array}\right]
$$

where $J(y)$ is the Jacobian matrix of the constraint equations and $H(y, \lambda)$ is $\nabla^{2} L(y, \lambda)$, the Hessian matrix of the Lagrangian function.

When the Lagrangian function is of order higher than quadratic, $W(y, \lambda)$ is no longer a constant matrix and the optimum points must be found by an iterative method by solving the following matrix equation iteratively:

$$
\left[\begin{array}{cc}
H(y, \lambda) & -J^{T}(y) \\
-J(y) & 0
\end{array}\right]\left[\begin{array}{l}
\Delta y \\
\Delta \lambda
\end{array}\right]=\left[\begin{array}{l}
-\nabla L(y) \\
-\nabla L(\lambda)
\end{array}\right]
$$

In order to solve for the correction vector, the numerical values of the matrix $W(y, \lambda)$ and the gradient vector $\nabla L(y, \lambda)$ have to be evaluated for a given value of $(y, \lambda)$. Both of these vectors have $(n+m)$ elements. Then the iterative solution at the $k^{\text {th }}$ iteration can be written as:

$$
\begin{aligned}
& W\left(z^{k}\right) \Delta z^{k+1}=-g\left(z^{k}\right) \\
& z^{k+1}=z^{k}+\Delta z^{k+1}
\end{aligned}
$$

\section{Transient Energy Function Method}

\subsection{Typical swing-equation}

A typical swing-equation includes the second-order differential equations associated with generator buses [18]:

$$
M_{i} \ddot{\delta}_{G i}+D_{i} \dot{\delta}_{G i}=P_{m i}-P_{G i} \quad i=1, \cdots, N
$$

where $\delta_{G i}$ is the rotor angle of $i^{\text {th }}$ generator, $P_{m i}$ and $P_{G i}$ are the mechanical power input and the electrical power output, respectively, $N$ is the number of generators, and $M_{i}$ and $D_{i}$ are, respectively, inertia and damping coefficients of the $i^{\text {th }}$ generator. Mechanical power input $P_{m i}$ is the prefault electrical power obtained in the power flow calculation. Electric power output is as follows:

$P_{G i}=\sum_{j=1}^{K}\left|V_{i}\right|\left|V_{j}\right|\left|Y_{i j}\right| \cos \left(\theta_{i j}-\delta_{G i}+\delta_{G j}\right)=0 \quad i=1, \cdots, N$ 
where $Y_{i j}$ is the reduced bus admittance matrix.

The criterion of transient stability is that the difference of any two rotor angles does not exceed the maximum secure relative swing angle, which is set as $180^{\circ}$, within a certain period after fault. Therefore, the transient stability is checked by using the generator angle difference:

$$
\left|\delta_{G i}(t)-\delta_{G j}(t)\right| \leq \delta_{G \max }
$$

where $\delta_{G i}(t)$ is the rotor angle of $i^{t h}$ generator at $t$, and $\delta_{G \max }$ is the maximum secure relative swing angle.

The process of transient stability analysis in (18) is very time consuming because of the use of Runge-Kutta method for simulation. Therefore, a faster process to check the transient stability is needed, by using the transient stability margin:

$$
\Delta v\left(=v_{\text {critical }}-v_{\text {transient }}\right)>0
$$

where $\Delta v, v_{\text {critical }}$ and $v_{\text {transient }}$ are transient stability energy margin, critical energy and transient energy, respectively.

\subsection{Transient stability energy margin}

In order to calculate energy margin, two values of the transient energy function have to be computed [18], [19]. The first value is transient energy that is the sum of kinetic and potential energy at the end of a fault. The second is critical energy that is potential energy at the controlling unstable equilibrium point (UEP). This paper used potential energy boundary surface (PEBS) method in determining the starting point. And the Davidon-FletherPowell (DFP) method for unconstrained minimization was used in calculating the exact UEP.

The transient energy margin is the difference between the transient energy and the critical energy. Energy margin function can be formulated by using the concept of the center of inertia (COI) reference frame. The energy margin function $(\Delta v)$ is defined as [19], [20]:

$$
\Delta v=v_{\text {critical }}-v_{\text {transient }}
$$

where the transient energy function and the critical energy function of (20) are defined as follows:

$$
\begin{aligned}
& v_{\text {transient }}=\frac{1}{2} \sum_{i=1}^{N} M_{i} \tilde{\omega}_{i}^{c 2}-\sum_{i=1}^{N} P_{i}\left(\theta_{i}^{c}-\theta_{i}^{s 1}\right) \\
& -\sum_{i=1}^{N-1} \sum_{j=i+1}^{N}\left[C_{i j}\left(\cos \theta_{i j}^{c}-\cos \theta_{i j}^{s 1}\right)\right. \\
& \left.+D_{i j} \frac{\theta_{i}^{c}+\theta_{j}^{c}-\theta_{i}^{s 1}-\theta_{j}^{s 1}}{\theta_{i j}^{c}-\theta_{i j}^{s 1}}\left(\sin \theta_{i j}^{c}-\sin \theta_{i j}^{s 1}\right)\right]
\end{aligned}
$$

$$
\begin{aligned}
& v_{\text {critical }}=-\sum_{i=1}^{N} P_{i}\left(\theta_{i}^{u}-\theta_{i}^{s 2}\right) \\
& -\sum_{i=1}^{N-1} \sum_{j=i+1}^{N}\left[C_{i j}\left(\cos \theta_{i j}^{u}-\cos \theta_{i j}^{s 2}\right)\right. \\
& \left.+D_{i j} \frac{\theta_{i}^{u}+\theta_{j}^{u}-\theta_{i}^{s 2}-\theta_{j}^{s 2}}{\theta_{i j}^{u}-\theta_{i j}^{s 2}}\left(\sin \theta_{i j}^{u}-\sin \theta_{i j}^{s 2}\right)\right]
\end{aligned}
$$

where

$$
\begin{aligned}
& C_{i j}=E_{i} E_{j} B_{i j}, D_{i j}=E_{i} E_{j} G_{i j} \text {, and for generator } i, \\
& P_{i}=P_{m i}-\left|E_{i}\right|^{2} G_{i i} \text {, } \\
& B_{i j}\left(G_{i j}\right) \text { : Transfer susceptance (conductance) in the } \\
& \text { reduced bus admittance matrix } \\
& \tilde{\omega}^{c 2} \quad: \text { Angular speed at fault clearing time } \\
& \theta^{c} \quad \text { : Angle at fault clearing time } \\
& \theta^{s 1} \quad \text { : Stable equilibrium point before fault } \\
& \theta^{u} \quad \text { : Unstable equilibrium point after fault clearing } \\
& \theta^{s 2} \quad \text { : Stable equilibrium point after fault clearing }
\end{aligned}
$$

The transient energy breaks out during the disturbance. So the longer the period of disturbance sustains, the more the transient energy occurs. As the critical energy is constant after disturbance, the energy margin will decrease to negative. For this reason the fault clearing time is one of the most important factors to determine the energy margin. Also, to apply in the real system, we consider the allotted power flow in the faulted line just before the fault because the load condition greatly affects the energy margin [20].

In order to compare the results of the transient energy margin and the time simulation two methods are applied to a 4-machine 6-bus system as shown in Fig. 1 [21, 22]. The load data, line and machine data for the system can be found in [21]. To check the transient stability with (18) the simulation is performed with fault clearing time of $0.41 \mathrm{sec}$ in (a) of Fig. 2. Also, the transient energy margin (19) is equal to 0.0553 , meaning the system is stable. But in (b) of Fig. 2 the simulation is performed with fault clearing time of 0.411 and the system is unstable. The transient energy

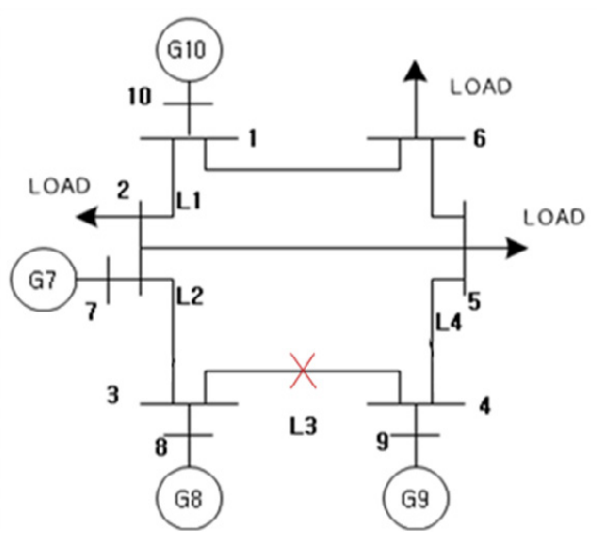

Fig. 1. The 4-machine 6-bus system. 


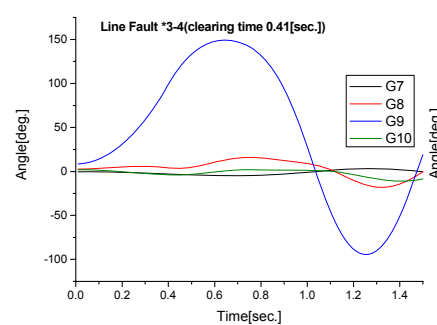

(a) clt $0.41 \mathrm{sec}$.

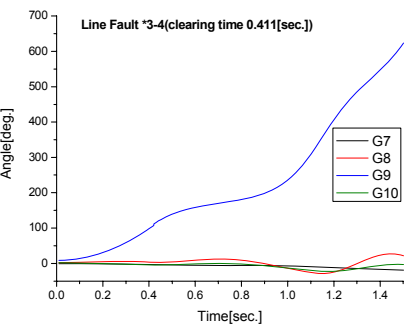

(b) clt $0.411 \mathrm{sec}$.
Fig. 2. Transient stability after line 3-4 fault.

margin in this case is equal to -0.0390 which is negative. From the two results, it is seen that the results of the time simulation and the transient energy margin agree to each other. Therefore, in order to check the transient stability the transient energy margin method can be used instead of the time simulation, such as the Runge-Kutta method.

\subsection{Energy margin sensitivity}

The transient energy margin is a function of parameters as follows:

$$
\Delta v=\Delta v\left(P_{m k}, \theta_{c r}, \theta_{c l}, E_{i}, G_{i j}, B_{i j}\right)
$$

where $P_{m k}$ is the mechanical power to the $k^{\text {th }}$ machine, $\theta_{c r}$ is the critical angle of the rotor, $\theta_{c l}$ is the rotor angle at fault clearing, $E_{i}$ is the constant voltage behind the direct axis transient reactance, and $G_{i j}, B_{i j}$ are respectively the transfer conductance and susceptance in the reduced bus admittance matrix.

A change in any of the functional parameters obviously causes a change in the energy margin. In general, for a given contingency, the sensitivity expression of the energy margin can be computed for any parameters as follows $[11,13]$ :

$$
\frac{\Delta(\Delta v)}{\Delta \alpha_{k}}=\sum_{k=1}^{n} \frac{\partial(\Delta v)}{\partial \alpha_{k}}
$$

where $\alpha_{k}$ is any system parameter of interest. For example, for generation rescheduling, the energy margin sensitivity can be evaluated for its change with respect to generation.

Two critical inferences can be made based on the value of the energy margin sensitivity. First, it is possible to rank the sensitivities and thereby determine the generators which will affect the energy margin most and hence affect the stability or instability of the system. Second, the sign of the sensitivity indicates the direction of the change in generation for a given change in the energy margin. The expression for energy margin sensitivity to change in generation is given as $[11,13]$ :

$$
\begin{aligned}
& \frac{\partial(\Delta v)}{\partial P_{m k}}=-M_{e q} M_{e q}^{c l} \frac{\partial \omega_{e q}^{c l}}{\partial P_{m k}}-\sum_{i=1}^{N}\left[P_{i}\left(\frac{\partial \theta_{i}^{u}}{\partial P_{m k}}-\frac{\partial \theta_{i}^{c l}}{\partial P_{m k}}\right)\right. \\
& +\left(\theta_{i}^{u}-\theta_{i}^{c l}\right) \frac{\partial P_{m i}}{\partial P_{m k}}+\sum_{i=1}^{N-1} \sum_{j=i+1}^{N}\left[\left(\frac{\theta_{i}^{u}+\theta_{j}^{u}-\theta_{i}^{c l}-\theta_{j}^{c l}}{\theta_{i j}^{u}-\theta_{i j}^{c l}} D_{i j} \cos \theta_{i j}^{u}\right.\right. \\
& \left.+C_{i j} \sin \theta_{i j}^{c l}\right)\left(\frac{\partial \theta_{i}^{u}}{\partial P_{m k}}-\frac{\partial \theta_{j}^{u}}{\partial P_{m k}}\right)-\left(\frac{\theta_{i}^{u}+\theta_{j}^{u}-\theta_{i}^{c l}-\theta_{j}^{c l}}{\theta_{i j}^{u}-\theta_{i j}^{c l}} D_{i j} \cos \theta_{i j}^{u}\right. \\
& \left.\left.+C_{i j} \sin \theta_{i j}^{c l}\right)\left(\frac{\partial \theta_{i}^{c l}}{\partial P_{m k}}-\frac{\partial \theta_{j}^{c l}}{\partial P_{m k}}\right)\right]+\sum_{i=1}^{N-1} \sum_{j=i+1}^{N} D_{i j}\left(\sin \theta_{i j}^{u}-\sin \theta_{i j}^{c l}\right) \\
& -2\left(\theta_{j}^{u}-\theta_{j}^{c l}\right) \\
& \left.\left(\theta_{i j}^{u}-\theta_{i j}^{c l}\right)^{2}\left(\frac{\partial \theta_{i}^{u}}{\partial P_{m k}}-\frac{\partial \theta_{i}^{c l}}{\partial P_{m k}}\right)+\frac{-2\left(\theta_{i}^{u}-\theta_{i}^{c l}\right)}{\left(\theta_{i j}^{u}-\theta_{i j}^{c l}\right)^{2}}\left(\frac{\partial \theta_{j}^{u}}{\partial P_{m k}}-\frac{\partial \theta_{j}^{c l}}{\partial P_{m k}}\right)\right]
\end{aligned}
$$

\section{Procedure of Generation Rescheduling}

Fig. 3 shows the flowchart for generation rescheduling to improve transient stability by using Newton's Approach. The detailed explanation for the procedure of Fig. 3 is as follows:

Step 1 : Calculate the transient energy margin (20) and transient energy margin sensitivity (25).

Step 2 : Determine the generation rescheduling priority based on the transient energy margin

Step 3 : Select the candidate generators that have the positive sensitivity for generation rescheduling except for slack generator.

Step 4 : Optimize the generation rescheduling by Newton' Approach (6).

Step 5 : Calculate the transient energy margin (20) from the generation rescheduling of step 4 .

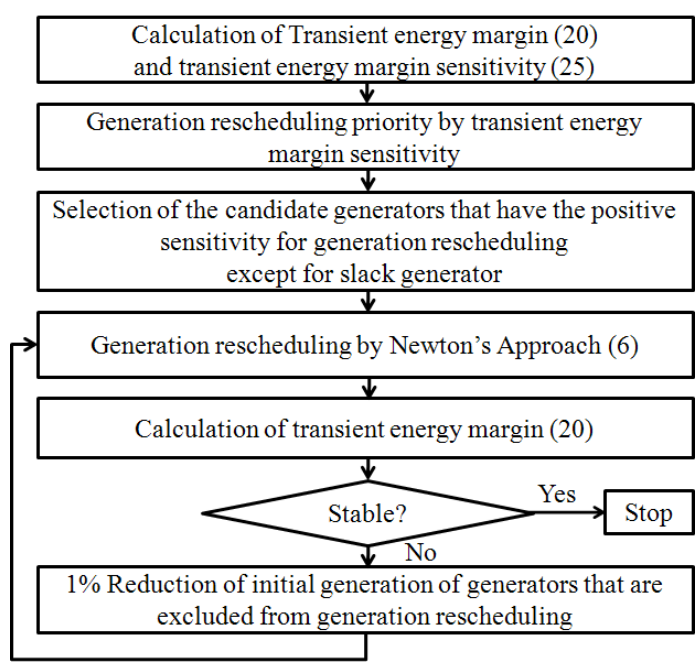

Fig. 3. Flowchart of generation rescheduling to improve transient stability 
Step 6 : If the system is stable, stop. Otherwise, if the system is not stable, initial generation of generators that are excluded from generation rescheduling is reduced by $1 \%$, respectively. Go to step 4 .

\section{Simulation Results And Discussion}

\subsection{Energy margin sensitivity indices and generation rescheduling results}

In order to improve transient stability with generation rescheduling based on the energy margin sensitivity, the three-phase fault is considered in transmission lines. The transient energy margin with each fault at clt $0.41 \mathrm{~s}$ shows in Table 1. Among the transient energy margin, the smallest is the margin of Line *3-4 fault, which can be used to improve transient stability. The fault at bus 3 is cleared by tripping the line at a tripping time, fault clearing time. The fuel cost and emission coefficients, and the generation limits are listed in Table 2.

Cases with and without considering energy margin sensitivity are simulated and compared to show that generation rescheduling can improve the transient stability

Table 1. Transient energy margin (clt $0.41 \mathrm{~s})$.

\begin{tabular}{cccc}
\hline Fault & $\begin{array}{c}\text { Critical } \\
\text { Energy(A) }\end{array}$ & $\begin{array}{c}\text { Transient } \\
\text { Energy(B) }\end{array}$ & $\begin{array}{c}\text { Transient Energy } \\
\text { Margin(A-B) }\end{array}$ \\
\hline Line *3 - 4 Fault & 1.5338 & 1.4784 & 0.0553 \\
Line *4 - 5 Fault & 1.2930 & 0.8697 & 0.4233 \\
Line *2 - 3 Fault & 1.8034 & 0.8543 & 0.9491 \\
Line 1 - *2 Fault & 1.7171 & 0.7122 & 1.0049 \\
Line *2 - 5 Fault & 2.2089 & 0.9487 & 1.2601 \\
\hline
\end{tabular}

if energy margin sensitivity is taken into account.

Case I represents the case that minimizes the fuel cost and emission $\left(\mathrm{SO}_{x}, N \mathrm{O}_{x}\right)$ for generation rescheduling of all generators, while not considering energy margin sensitivity.

Case II represents the case that minimizes the fuel cost and emission $\left(\mathrm{SO}_{x}, N \mathrm{~N}_{x}\right)$ for generation rescheduling of those generators that are selected with the energy margin sensitivity based on Fig. 3 .

Table 3 shows the results of Case I. It is seen that the proposed approach can explore the solutions of a multiobjective optimization problem. However the energy margins of each solution in the fault at bus 3 are negative value for some margins. This means the energy margin sensitivity is not considered while optimizing generation rescheduling. The rotor angle plots with fault clearing time of each fault after generation rescheduling are shown in (a)-(c) of Fig. 4.

Table 4 shows the results of Case II. The energy margin sensitivity indices obtained by using (25) are indicated in second column of Table 4. In case of the fault at bus 3, machines 7, 8 and 10 are selected and in case of the fault at bus 2 , machines 7 and 10 are selected as the candidate generators for generation rescheduling. The proposed approach explores the solutions of a multiobjective optimization problem. Also the energy margins of each solution in the fault at bus 3 are positive value, which means the system is stable. The energy margin sensitivity is considered while optimizing generation rescheduling. If the system is still unstable after generation rescheduling, the initial generation has to be reduced in Step 6 of Section IV and Fig. 3. The rotor angle plots with fault clearing time at fault after generation rescheduling are shown in (d)-(f) of Fig. 4.

Also Table 4 and Fig. 5 show the results of the fault at

Table 2. Transient energy margin, fuel cost and emission coefficients, and generation limits.

\begin{tabular}{|c|c|c|c|c|c|c|c|c|c|c|}
\hline \multirow{2}{*}{ Unit No. } & \multicolumn{3}{|c|}{ Fuel Cost Coefficients } & \multicolumn{5}{|c|}{ Emission Coefficients } & \multicolumn{2}{|c|}{ Unit Limit [MW] } \\
\hline & $a_{i}$ & $b_{i}$ & $c_{i}$ & $\alpha_{i}$ & $\beta_{i}$ & $\gamma_{i}$ & $\zeta_{i}$ & $\mu_{i}$ & $P_{i}^{\min }$ & $P_{i}^{\max }$ \\
\hline 7 & 20.0 & 180.0 & 40.0 & 4.258 & -5.094 & 4.586 & $1.0 \mathrm{E}-6$ & 8.000 & 10 & 200 \\
\hline 8 & 10.0 & 200.0 & 100.0 & 4.091 & -5.543 & 6.490 & $2.0 \mathrm{E}-4$ & 2.857 & 10 & 200 \\
\hline 9 & 10.0 & 100.0 & 60.0 & 5.326 & -3.550 & 3.380 & $2.0 \mathrm{E}-3$ & 2.000 & 10 & 200 \\
\hline 10 & 10.0 & 150.0 & 100.0 & 6.131 & -5.555 & 5.151 & $1.0 \mathrm{E}-5$ & 6.667 & 10 & 200 \\
\hline
\end{tabular}

Table 3. Solution and energy margin of generation re-scheduling for all generators.

\begin{tabular}{|c|c|c|c|c|c|}
\hline Unit. No. & & Initial Gen. [MW] & Fuel Cost Minimization[MW] & Emission Minimization [MW] & Compro-mise Solution [MW] \\
\hline 7 & & 63.2 & 26.56 & 37.64 & 34.34 \\
\hline 8 & & 10.0 & 10.0 & 29.23 & 16.83 \\
\hline 9 & & 30.0 & 67.79 & 20.39 & 43.14 \\
\hline 10 & & 20.0 & 22.28 & 37.56 & 30.58 \\
\hline \multicolumn{6}{|c|}{ Three-phase fault at bus 3 , Line cleared $* 3-4$} \\
\hline \multirow{2}{*}{ EnergyMargin } & $\mathrm{t} 0.41 \mathrm{~s}$ & 0.0553 & -1.3951 & 1.0656 & -0.9906 \\
\hline & $\mathrm{t} 0.411 \mathrm{~s}$ & -0.0390 & -1.2572 & 1.0064 & -1.0654 \\
\hline \multicolumn{6}{|c|}{ Three-phase fault at bus 2 , Line cleared $* 2-3$} \\
\hline \multirow{2}{*}{ EnergyMargin } & $\mathrm{t} 0.54 \mathrm{~s}$ & 0.0436 & -0.9994 & 0.5810 & -0.7779 \\
\hline & $\mathrm{t} 0.541 \mathrm{~s}$ & -0.0218 & -1.0783 & 0.6051 & -0.7563 \\
\hline \multirow{2}{*}{\multicolumn{3}{|c|}{$\begin{array}{l}\text { Fuel Cost }(\$ / \mathrm{h}) \\
\text { Emission }(\text { ton } / \mathrm{h})\end{array}$}} & 255.35 & 283.78 & 262.59 \\
\hline & & & 0.1726 & 0.1589 & 0.1624 \\
\hline
\end{tabular}




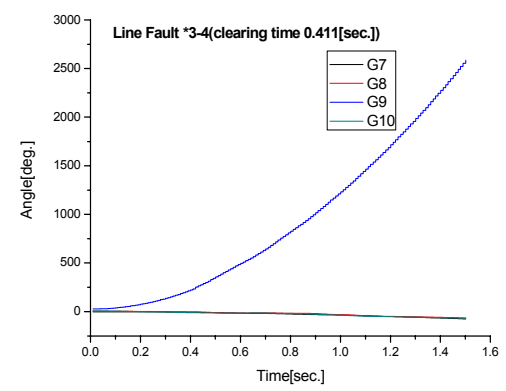

(a) Fuel cost min. of Case I.

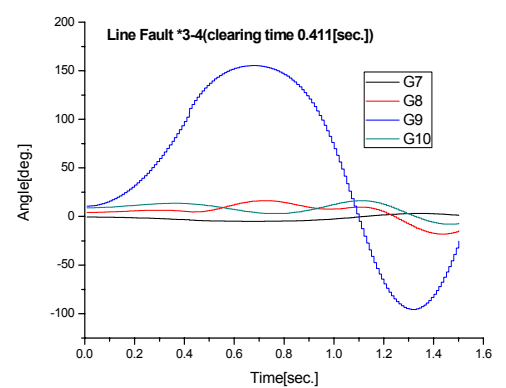

(d) Fuel cost min. of Case II.

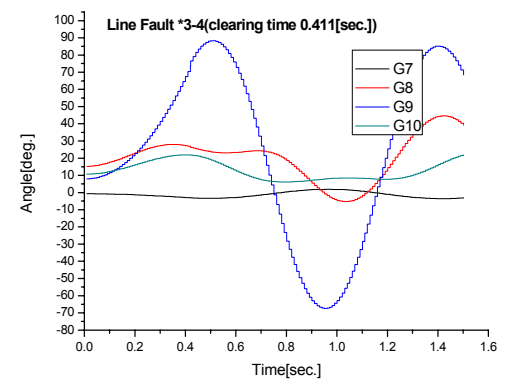

(b) Emission min. of Case I.

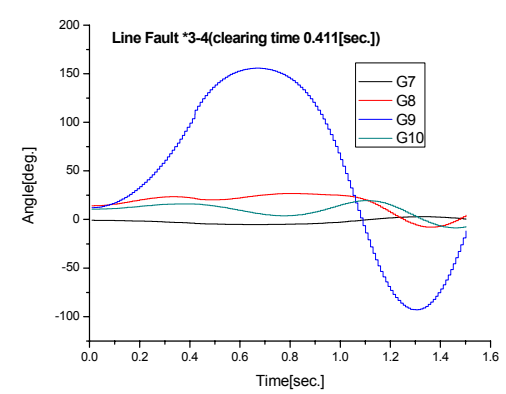

(e) Emission min. of Case II.

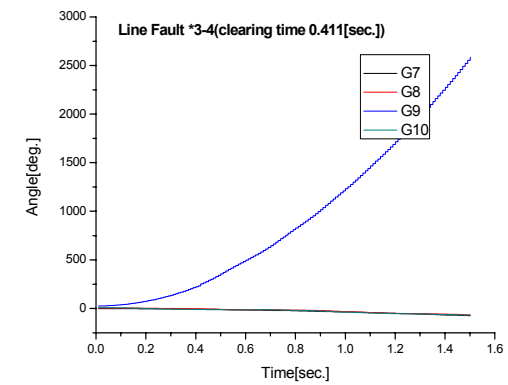

(c) Compromise solution min. of Case I.

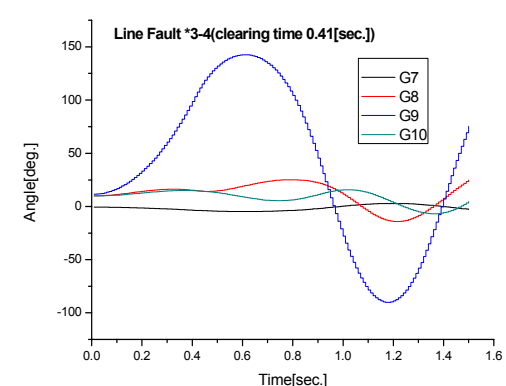

(f) Compromise solution min. of Case II.

Fig. 4. Rotor angle plots (Fault at bus 3, line cleared *3-4).

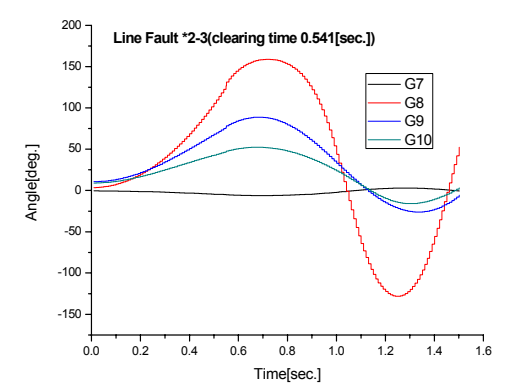

(a) Fuel cost min. of Case I.

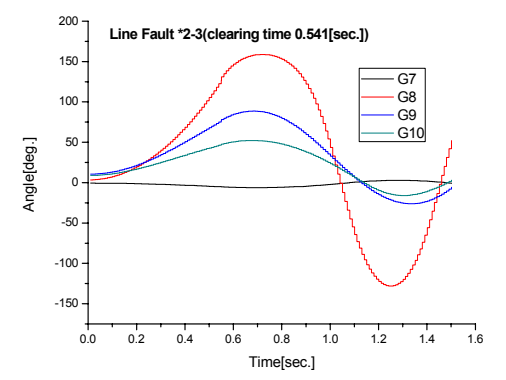

(d) Fuel cost min. of Case II.

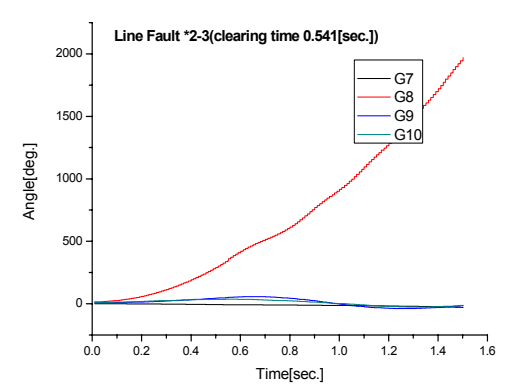

(b) Emission min. of Case I.

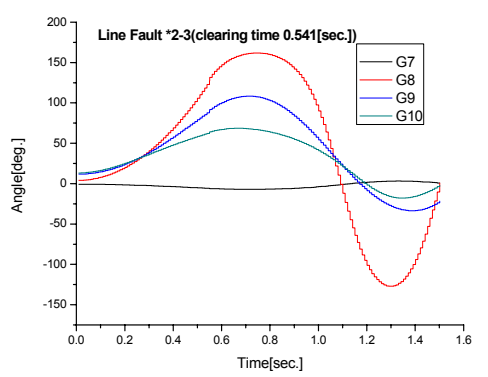

(e) Emission min. of Case II.

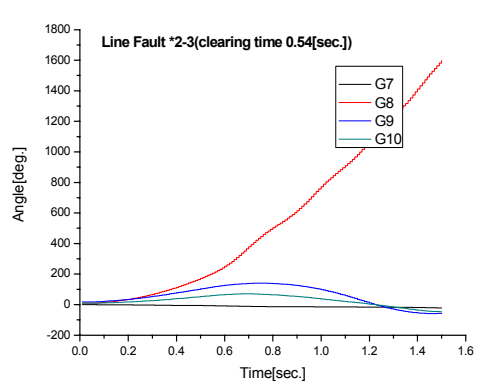

(c) Compromise solution min. of Case I.

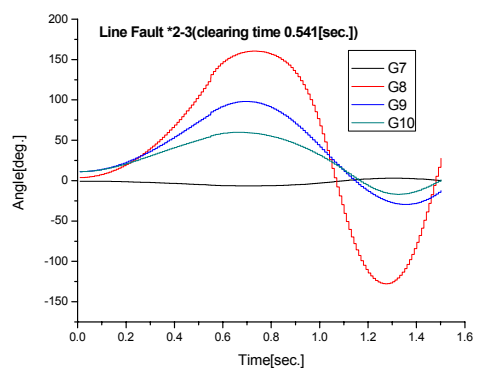

(f) Compromise solution min. of Case II.

Fig. 5. Rotor angle plots (Fault at bus 2, line cleared *2-3).

bus 2 and are similar to the results of the fault at bus 3 . Therefore it is seen that the proposed approach can explore the solutions of the multiobjective optimization problem and improve the transient stability of system.

To demonstrate the effectiveness of the proposed approach, recall that the multiobjective optimization problem (6) is converted to an unconstrained single objective optimization problem by using the Lagrangian function (7). In (7) $w$ is a weight factor in the range from 0 to 1 . The value $w=1.0$ implies the minimum operating cost and the solution of the fuel cost minimization is an extreme point in the trade-off surface. As the importance of the emission reduction increases $(w>0)$ then the optimum solution will move toward the emission reduction. The 


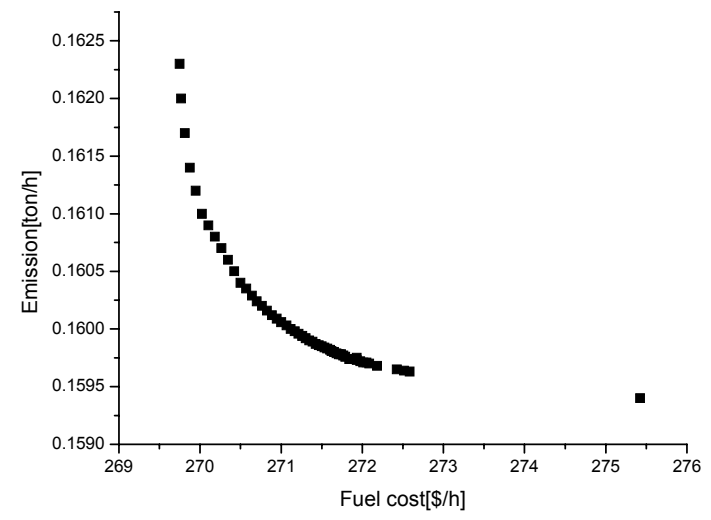

Fig. 6. Pareto optimal solution of fuel cost vs. emission.

value $w=0.0$ implies minimum emission and the solution of the emission minimization is another extreme point in the trade-off surface. The relationship of fuel cost and emission cost as $w$ varies is shown in Fig. 6. This problem can be also solved as a multiobjective optimization problem where both fuel cost and emission were optimized simultaneously. This results in the Pareto optimization, where the trade-off curve or Pareto front shows the relationship of the non-dominated solutions in the fuel cost and emission cost. It can be seen that the Pareto optimum has a number of non-dominated solutions and the proposed approach can explore more efficient and non-inferior solutions of a multiobjective optimization problem.

\section{Conclusion}

This paper presents a generation rescheduling method for the enhancement of transient stability in power systems. In order to determine the generation rescheduling priority the energy margin sensitivity is calculated. The candidate generators for the generation rescheduling are determined by the energy margin sensitivity. The generation rescheduling formulates the Lagrangian function with the fuel cost function and emission cost of generators. The generation rescheduling searches for the solution of the Lagrangian function by using the Newton's approach. The Pareto optimum in the fuel cost and emission cost has a number of non-dominated solutions. The proposed approach can explore more efficient and non-inferior solutions of a multiobjective optimization problem and improve the transient stability of system.

\section{Acknowledgements}

This research was supported by Korea Electric Power Corporation Research Institute through Korea Electrical Engineering \& Science Research Institute. [Grant number : R13TA18]

\section{References}

[1] H. W. Dommel and W. F. Tinney, "Optimal power flow solution," IEEE Trans. on PAS, 87, pp. 18661876, 1968.

[2] D. I. Sun, B. Ashley, B. Brewer, A. Hughes, and W. F. Tinney, "Optimal power flow by the Newton's Approach," IEEE Trans. on PAS, Vol. 103, No. 10, pp. 2864-2880, 1984.

[3] R. Y. Kwok, "Optimal power flow in power system analysis," Energy Systems Research Center, The University of Texas at Arlington, 1987.

[4] C. W. Sanders and C. A. Monroe, "An Algorithm for Real-Time Security Constrained Economic Dispatch," IEEE PWRS-2, Nov. 1987.

[5] A. Monticelli, M.V.F. Pereira, and S. Granville, "Security-constrained optimal power flow with post contingency corrective rescheduling," IEEE PWRS-2, Feb. 1988.

[6] W. C. Merrit, C. H. Saylor, R. C. Burchett and H. H. Happ, "Security constrained optimization-a case study," IEEE PWRS-3, Aug. 1988.

[7] J. Sterling, M. A. Pai, and P. W. Sauer, "A Methodology to secure and optimal operation of a power system for dynamic contingencies," Journal of Electric Machines and Power Systems, Vo1. 19, No. 5, Sept.Oct. 1991.

[8] A. A. Fouad and J. Tong, "Stability constrained optimal rescheduling of generation," IEEE Trans. on Power Systems, Vol. 8, No. 1, pp.105-112, Feb. 1993.

[9] D. H. Kuo and A. Bose, "A generation rescheduling method to increase the dynamic security of power systems," IEEE Transactions on Power Systems, Vol. 10, No. 1, pp. 68-76, Feb. 1995.

[10] P. W. Sauer, K. D. Demaree, and M. A. Pai, "Stability limited load supply and interchange capability," IEEE Trans. on Power Apparatus and Systems, Vol. 102, No. 11, pp. 3637-3643, Nov. 1983.

[11] V. Vittal, E. Zhou, C. Hwang, and A. A. Fouad, "Derivation stability limits using analytical sensitivity of the transient energy margin," IEEE Trans. on Power Systems. Vol. 4, No. 10, pp. 1363-1372, Oct. 1989.

[12] J. Tong, H. D. Chiang, and T. P. Conneen, "A sensitivity-based BCU method for fast derivation of stability limits in electric power systems," $92 \mathrm{WM}$ 149-5 PWRS.

[13] J. A. Momoh and C. B. Fffiong, "Generation rescheduling for dynamic security enhancement for multi-area power system," Proc. IEEE Int. Conf. Computational Cybernetics and Simulation: Systems, Man and Cybernetics, 4, pp. 3437-3442, 1987.

[14] D. Z. Fang, Y. Xiaodong, S. Jingqiang, Y. Shiqiang, and $\mathrm{Z}$. Yao, "An optimal generation rescheduling approach for transient stability enhancement," IEEE Trans. on Power System, Vol. 22, No. 1, pp. 386-394, Feb. 2007. 
[15] J. H. Talaq, F. El-Hawary, and M. E. El-Hawary, “A summary of environmental / economic dispatch algorithms," IEEE Trans. on Power Syst., Vol. 9, pp. 15081516, Aug. 1994.

[16] M. A. Abido, "Environmental / economic power dispatch using multiobjective evolutionary algorithms," IEEE Trans. on Power System, Vol. 18, No. 4, pp. 1529 -1537, Nov. 2003.

[17] L. H. Wua, Y. N. Wanga, X. F. Yuana, and S. W. Zhoub, "Environmental/economic power dispatch problem using multi-objective differential evolution algorithm," Electric Power Systems Research Vol. 80, pp. 11711181, 2010.

[18] M. A. Pai, Energy Function Analysis for Power System Stability, Kluwer Academic Publishers, 1989.

[19] A. A. Fouad and V. Vittal, "Power system transient stability analysis using the transient energy function methods," Prentice Hall, 138, 1992.

[20] S.-N. Kim, K.-H. Kim, and S.-K. You, "Real-time estimation of multiple series capacitor quantity for transient stability energy margin improvement," IEEE PES Winter Meeting, pp. 238-243, Jan. 2002.

[21] K.-H. Kim, S.-N. Kim, S.-B. Rhee, S.-K. Lee, and K.-B. Song, "Assessment of total transfer capability subject to transient stability energy margin," IEEE Transmission and Distribution Asia, September, pp. 238-243, 2008.

[22] K.-H. Kim, S.-B. Rhee, K.-B. Song, K.-J. Hwang, and K. Y. Lee, "Optimal power flow based on generation rescheduling priority for transient stability enhancement," IFAC Symposium on Power Plants and Power System Control, Toulouse, France, Sep. 2012.

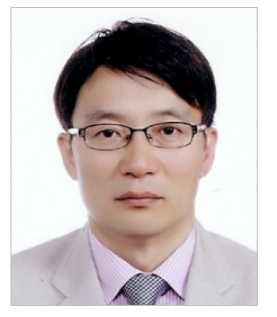

Kyu-Ho Kim received his B.S., M.S. and Ph.D. degrees from Hanyang University, Korea, in 1988, 1990 and 1996, respectively. He is an Associate Professor in the Department of Electrical Engineering at Hankyong University, Korea. He was a Visiting Scholar at Baylor University for 2011-2012. His research interests include power system control and operation, optimal power flow and evolutionary computation.

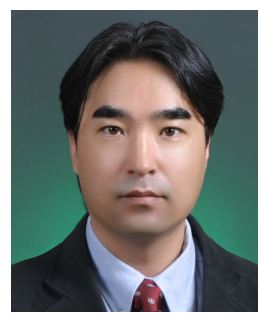

Sang-Bong Rhee received his B.S., M.S. and Ph.D. degrees from Hanyang University, Korea, in 1994, 1999 and 2004, respectively. He is currently an Assistant Professor in the Department of Electrical Engineering at Yeungnam University, Korea. His research interests include artificial intelligence applica- tions, distribution power system analysis, operation, and control.

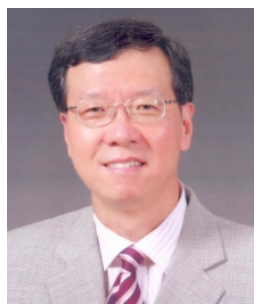

Kab-Ju Hwang received the B.S. degree in Electrical Engineering from the University of Ulsan, Korea, in 1975 and M.S. and Ph.D. degrees in Electrical Engineering from Hanyang University, Korea, in 1977 and 1983, respectively. He joined the faculty of the Department of Electrical Engineering, the University of Ulsan in 1983 . He is currently a Professor in the Department of Electrical Engineering, the University of Ulsan. He is a member of the Korea Institute of Electrical Engineers and IEEE. His fields of interest are in power system operation and planning.

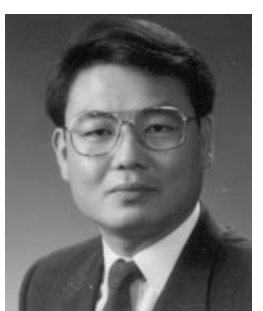

Kyung-Bin Song received his B.S. and M.S. degrees in Electrical Engineering from Yonsei University, Korea, in 1986 and 1988, respectively. He received his $\mathrm{Ph} . \mathrm{D}$. degree in Electrical Engineering from Texas A\&M University, College Station, Texas in 1995. He is currently an Associate Professor in Electrical Engineering at Soongsil University, Seoul, Korea. His research interests include power system operation and control, power system economics, the optimization of the large scale systems, and the fuzzy system and its applications.

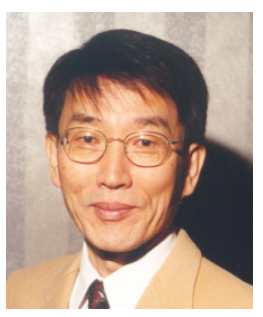

Kwang Y. Lee received the B.S. degree in Electrical Engineering in 1964 from Seoul National University, M.S. degree in Electrical Engineering in 1968 from North Dakota State University, and Ph. D. degree in Systems Science in 1971 from Michigan State University. He was elected as a Fellow of IEEE in the January 2001 for his contributions to the development and implementation of intelligent system techniques for power plants and power systems control and as a Life Fellow of IEEE since January 2008. He has been working in the area of power plants and power systems control for over thirty years at Michigan State, Oregon State, University of Houston, the Pennsylvania State University, and the Baylor University, where he is Professor and Chairman of the Department of Electrical and Computer Engineering. His research interests include control, operation, and planning of power and energy systems; computational intelligence, intelligent control and their applications to power and energy systems, and modeling, simulation and control of micro-grids with renewable and distributed energy sources. 